\title{
Tidal disruption of stars by supermassive black holes: The X-ray view
}

\section{S. Komossa ${ }^{a}$}

\author{
Max-Planck-Institut für Radioastronomie, Auf dem Hügel 69, 53121 Bonn, Germany
}

\begin{abstract}
The tidal disruption of stars by supermassive black holes produces luminous soft X-ray accretion flares in otherwise inactive galaxies. First events have been discovered in X-rays with the ROSAT observatory, and have more recently been detected with XMM-Newton, Chandra and Swift, and at other wavelengths. In X-rays, they typically appear as very soft, exceptionally luminous outbursts of radiation, which decline consistent with $L \propto t^{-5 / 3}$ on the timescale of months to years. They reach total amplitudes of decline up to factors 1000-6000 more than a decade after their initial high-states, and in low-state, their host galaxies are essentially X-ray inactive, optically inactive, and radio inactive. X-ray luminous tidal disruption events (TDEs) represent a powerful new probe of accretion physics near the event horizon, and of relativistic effects. TDEs offer a new way of estimating black hole spin, and they are signposts of supermassive binary black holes and recoiling black holes. Once discovered in the thousands in upcoming sky surveys, their rates will probe stellar dynamics in distant galaxies, and they will uncover the so far elusive - population of intermediate mass black holes in the universe, if they do exist. Further, the reprocessing of the flare into IR, optical and UV emission lines provides us with multiple new diagnostics of the properties of any gaseous material in the vicinity of the black hole (including the disrupted star itself) and in the host galaxy. First candidate events of this kind have been reported recently.
\end{abstract}

\section{INTRODUCTION}

The tidal disruption, and subsequent accretion, of a star by a supermassive black hole $(\mathrm{SMBH})$ produces a luminous flare of electromagnetic radiation, visible out to large cosmic distances (Luminet 1985, Rees 1988). Events have initially been discovered in the form of luminous $\mathrm{X}$-ray flares from otherwise non-active galaxies during the ROSAT all-sky survey (e.g., Komossa \& Bade 1999), providing us with a new method of unveiling the dormant SMBH population in the universe, and studying accretion processes and the immediate environment of SMBHs. More recently, events from otherwise quiescent galaxies have also been seen in the UV, optical, and radio regime, and they continue to be detected in X-rays. Upcoming sky surveys will detect these events by the 1000s, enabling a wealth of new studies and applications.

A star approaching a SMBH is subject to strong tidal forces exerted by the black hole. Once these exceed the self-gravity of the star, the star is disrupted (Hills 1975). The distance at which this happens, the tidal radius, is given by

$$
r_{\mathrm{t}} \simeq 710^{12}\left(\frac{M_{\mathrm{BH}}}{10^{6} M_{\odot}}\right)^{\frac{1}{3}}\left(\frac{M_{*}}{M_{\odot}}\right)^{-\frac{1}{3}} \frac{r_{*}}{r_{\odot}} \mathrm{cm} .
$$

A fraction of the stellar material will be on unbound orbits and escape, while the rest will eventually be accreted. In the course of the whole process, including the disruption, ejection and accretion of the stellar material, there are several sites and sources of radiation (Fig. 1):

- After disruption, a fraction of the stellar material is accreted onto the SMBH. This produces a flare of electromagnetic radiation, peaked in the EUV

a e-mail: skomossa@mpifr.de or soft X-rays, which can be as bright as the Eddington luminosity. It will last on the order of weeks, followed by a decline on the timescale of months to years. In X-rays, the decline law scales as $L \propto t^{-5 / 3}$. In most cases, this accretion phase is the most luminous phase of the tidal disruption event (TDE) (e.g., Rees 1988).

- During the accretion phase, a jet may be launched (e.g., Cheng et al. 2006).

- If the stellar orbit is deeply plunging, the squeezing of the star can be extreme. If nuclear burning is ignited during this process, this would result in a GRB-like explosion (e.g., Luminet \& Marck 1985, Carter \& Luminet 1982).

- Part of the disrupted star will be on unbound trajectories. Its collision with surrounding interstellar matter may produce shocks like in a supernova remnant (Khokhlov \& Melia 1996).

- Stream-stream collisions of the returning stellar matter with more tightly bound material, will be another source of shocks (e.g., Kim et al. 1999).

- Compact objects spiralling into the SMBH, like white dwarfs, are a strong source of gravitational waves (GW) which will be detectable with future space-based GW observatories (e.g., Amaro-Seoane et al. 2007).

- The luminous continuum emission produced during the accretion phase of TDEs photoionizes ambient gaseous material and will be reprocessed into IRoptical-UV emission lines, causing an emissionline "light-echo" which is sensitive to the geometry and properties of the surrounding medium (e.g., Komossa et al. 2008).

Most or all TDEs observed so far have been identified during their bright accretion phase. This review focusses 


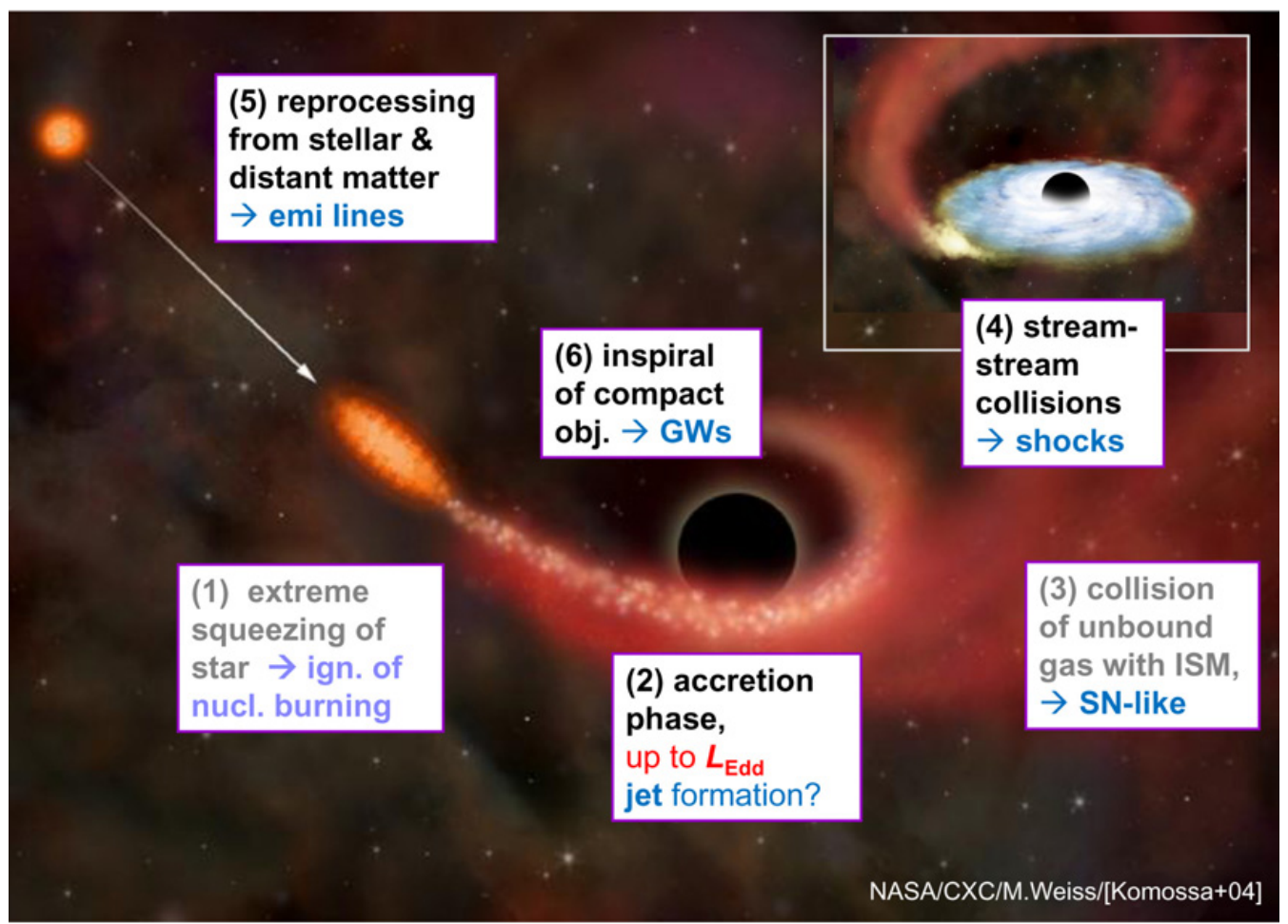

Figure 1. Sites and sources of radiation during the tidal disruption of a star by a supermassive black hole. The brightest phase is the accretion phase.

on observations and implications of TDEs discovered in $\mathrm{X}$-rays, and the accompanying emission-line signatures induced by a photoionizing EUV-soft X-ray spectrum. Observations of UV and optical TDEs ${ }^{1}$ will be reviewed by Gezari (these proceedings).

\section{TIDAL DISRUPTION EVENTS IN X-RAYS}

\subsection{General properties}

The predicted tidal disruption event rates are low, on the order of $10^{-4 \ldots-5} / \mathrm{yr} / \mathrm{galaxy}$. Therefore, large-area or allsky survey type experiments are best suited to detect these events. Since the accretion phase produces a luminous flare, with peak in the EUV or soft X-rays, TDEs are best detected in X-rays. Further, in X-rays the contrast with respect to the (faint, extended) permanent emission of the host galaxy is highest, and X-rays trace the immediate vicinity of the black hole, opening up new possibilities of probing accretion near the event horizon and relativistic effects (Sect. 3).

The ROSAT observatory, with its soft X-ray sensitivity, all-sky coverage and long lifetime (Trümper 2001), was ideally suited for detection of TDEs. Indeed, events were first discovered with ROSAT (Bade et al. 1996, Komossa \& Bade 1999, Komossa \& Greiner 1999, Grupe et al. 1999, Greiner et al. 2000). Two of these, NGC 5905 and RXJ1242-1119, continue to be the bestmonitored events in terms of their long-term X-ray

\footnotetext{
${ }^{1}$ e.g., Gezari et al. (2006), Komossa et al. (2008), van Velzen et al. (2011a), Cenko et al. (2012a).
}

lightcurves, spanning time intervals of more than a decade (Komossa et al. 2004, Halpern et al. 2004, Komossa 2004). More recently, similar X-ray events have been found with Chandra and XMM-Newton (Esquej et al. 2007 and 2008, Cappelluti et al. 2009, Maksym et al. 2010, Lin et al. 2011, Saxton et al. 2012), and two unusual sources with Swift (e.g., Burrows et al. 2011, Bloom et al. 2011, Zauderer et al. 2011, Levan et al. 2011, Barres de Almeida \& De Angelis 2011, Cenko et al. 2012b). Essentially all of the soft X-ray events share similar properties, and in particular:

- Huge observed (soft) X-ray peak luminosities, reaching almost up to $10^{45} \mathrm{erg} / \mathrm{s}$.

- Amplitudes of decline up to factors 1000-6000, more than a decade after their observed high-states.

- Very soft X-ray spectra during the high-states $\left(k T_{\mathrm{BB}} \sim 0.04-0.1 \mathrm{keV}\right)$, followed by a spectral hardening on the time scale of years.

- Their host galaxies show essentially no evidence for permanent activity as it is seen in active galactic nuclei (AGN). Years after the flare, the galaxies are optically inactive, radio inactive, and $\mathrm{X}$-ray inactive.

- X-ray lightcurves are consistent with a rapid onset of the flare (in the few cases, where pre-flare observations exist), then a decline on the timescale of months-years, consistent with the law $L \propto t^{-5 / 3}$ predicted by tidal disruption theory.

- SMBH masses of the host galaxies are on the order of $10^{6}-10^{8} \mathrm{M}_{\odot}$.

- TDE rates derived from X-ray surveys are on the order of $10^{-4}-10^{-5} / \mathrm{yr} /$ galaxy. 


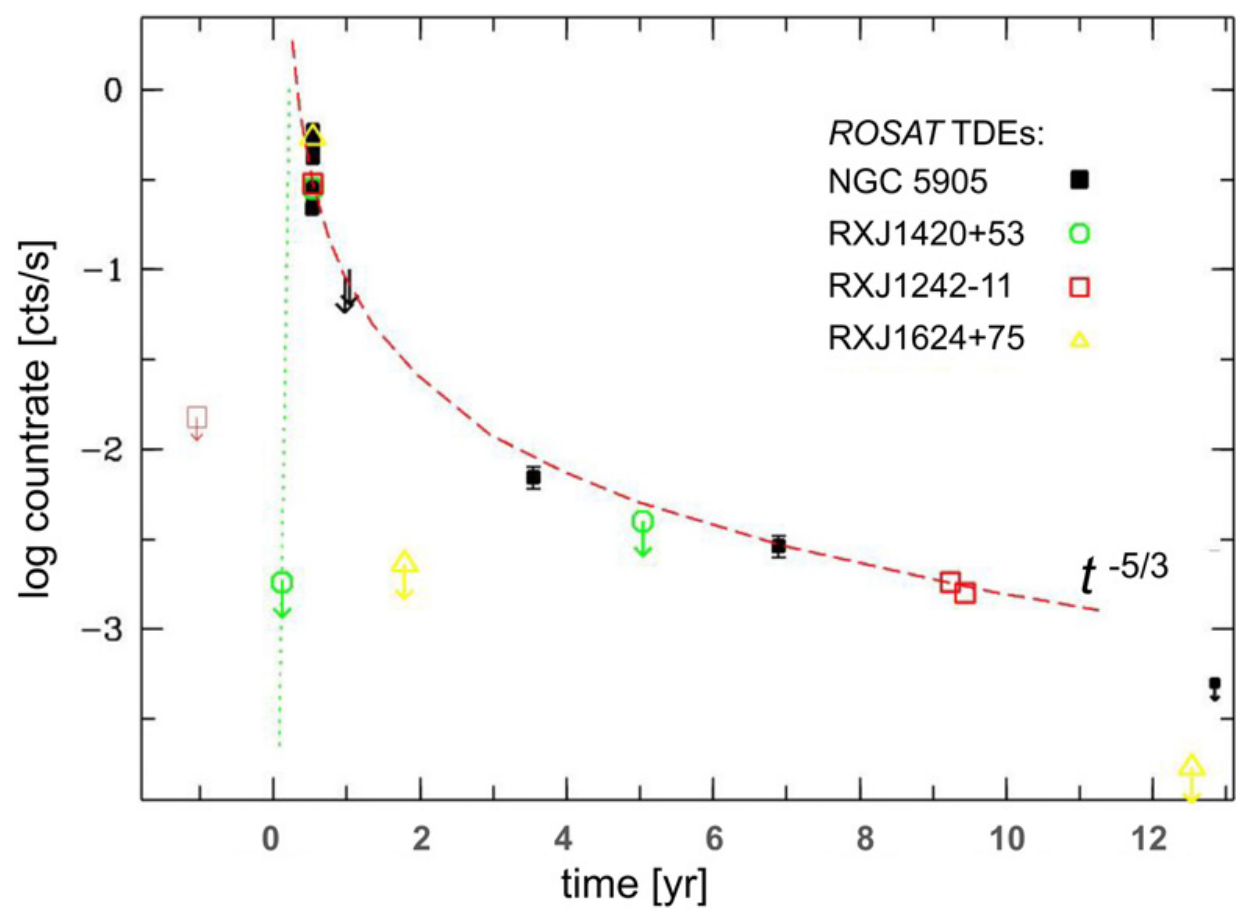

Figure 2. Joint X-ray lightcurve of the initial ROSAT events, all shifted to the same high-state time. The decline is well consistent with the predicted $t^{-5 / 3}$ law (dashed lined). This point was first made based on ROSAT data of NGC5905 (Komossa \& Bade 1999), and later for the overall luminosity evolution of the sources displayed above (e.g., Fig. 1 of Komossa 2004).

All these properties agree remarkably well with orderof-magnitude predictions from tidal disruption theory (e.g., Rees 1988, 1990).

Computations of the stellar evolution, from the early squeezing of the star, the actual disruption, ejection and accretion of material, and up to the late stages of the accretion phase, are very challenging. Based on substantial progress during the last several years, improved and new predictions for the lightcurve evolution, and other observable properties of TDEs, have been made (e.g., Brassart \& Luminet 2010, Strubbe \& Quataert 2011, Lodato et al. 2009, Lodato \& Rossi 2011, van Velzen et al. 2011b, Stone et al. 2012, Guillochon \& RamirezRuiz 2012, Hayasaki et al. 2012, Kesden 2012). In particular, these generally confirm the overall X-ray decline law, $L \propto t^{-5 / 3}$, consistent with the ROSAT flares (e.g., Komossa \& Bade 1999; see Fig. 2 above). They also point to important deviations in early and late phases of the lightcurve evolution, and in non-X-ray bands, and therefore provide us with new tools of studying the physics of tidal disruption, and deriving the type of disrupted star and the spin of the SMBH.

\subsection{Notes on individual events}

\subsubsection{NGC 5905}

NGC 5905 underwent a giant, ultrasoft $(k T=0.06 \mathrm{keV})$ and luminous $\left(L_{\mathrm{x}, \text { peak }}=710^{42} \mathrm{erg} / \mathrm{s}\right)$ X-ray outburst during the ROSAT all-sky survey (RASS) in 1990. It remained bright for at least $\sim 5$ days (the time interval it was repeatedly covered during the RASS,

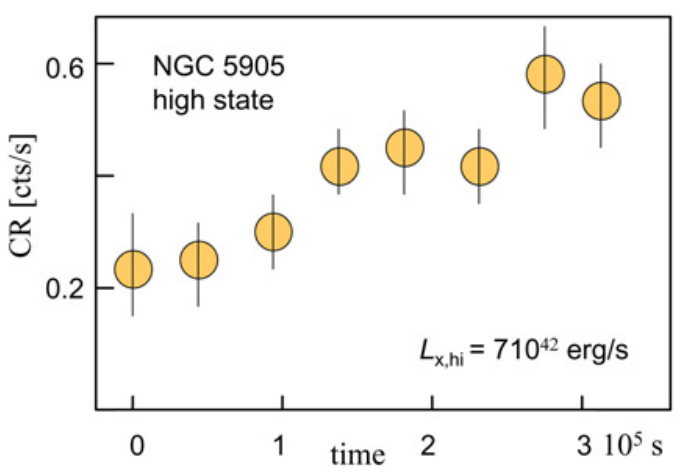

Figure 3. ROSAT high-state lightcurve of NGC 5905, observed during the RASS. It shows a rise within several days toward the observed peak of $L_{\mathrm{sx}}=710^{42} \mathrm{erg} / \mathrm{s}$.

Fig. 3) increasing in luminosity to the observed peak, then declined on the timescale of month to years (Fig. 2). All observations of this event are in very good agreement with tidal disruption theory (Komossa \& Bade 1999).

Within the errors, the X-rays came from the center of this nearby $(z=0.011)$, giant, barred spiral galaxy. While the flare X-ray spectrum was initially very soft, it had hardened significantly $\left(\Gamma_{\mathrm{x}}=-2.4\right) 3$ years later, when reobserved with ROSAT. The decline of the X-ray lightcurve is well consistent with the expected $t^{-5 / 3}$ law, as first reported based on its ROSAT observations (Komossa \& Bade 1999) and confirmed with Chandra (Halpern et al. 2004).

Whenever enough data exist, the ROSAT events, and most of the more recent events, follow a similar trend in spectral and lightcurve evolution as NGC 5905, providing 
Table 1. X-ray TDE candidates in quiescent galaxies.

\begin{tabular}{llll}
\hline $\begin{array}{l}\text { TDE or host galaxy } \\
\text { name }\end{array}$ & redshift & identified with & reference \\
\hline NGC 5905 & & & \\
RXJ1242-1119 & 0.011 & ROSAT & Bade et al. 1996, Komossa \& Bade 1999 \\
RXJ1624+7554 & 0.050 & ROSAT & Komossa \& Greiner 1999, Komossa et al. 2004 \\
RXJ1420+5334 & 0.147 & ROSAT & Grupe et al. 1999 \\
NGC 3599 & 0.0028 & XMM-Newton & Esquej et al. 2007, 2008 \\
SDSSJ1323+4827 & 0.087 & XMM-Newton & Esquej et al. 2007, 2008 \\
TDXFJ1347-3254 & 0.037 & ROSAT & Cappelluti et al. 2009 \\
SDSS J1311-0123 & 0.195 & Chandra & Maksym et al. 2010 \\
2XMMi J1847-6317 & 0.035 & XMM-Newton & Lin et al. 2011 \\
SwiftJ1644+57 & 0.354 & Swift & Burrows et al. 2011, Bloom et al. 2011 \\
SDSSJ1201+3003 & 0.146 & XMM-Newton & Saxton et al. 2012 \\
SwiftJ2058.4+0516 & 1.185 & Swift & Cenko et al. 2012b \\
\hline
\end{tabular}

independent evidence that the same mechanism was at work in all these cases.

In order to test, whether TDEs do launch radio jets, radio follow-ups of NGC 5905 were performed with the VLA (Komossa 2002) 6 years after the initial outburst. No radio emisson was detected $(f<0.15 \mathrm{mJy}$ at $8.46 \mathrm{GHz}$; see Sect. 2.3 of Komossa 2002 for an extended discussion of the radio properties of NGC 5905).

Ground-based optical spectroscopy of NGC 5905 (Komossa \& Bade 1999) after the flare, confirmed an HII-type galaxy spectrum. While the HST spectrum of NGC 5905 shows faint $[\mathrm{OIII}] / \mathrm{H} \beta>3$ from the central region (Gezari et al. 2003) which plausibly has been excited by the TDE itself, two other ROSAT events, RXJ1242-1119 and RXJ1624+7554, show just an absorption spectrum of a gas-poor host galaxy, with no emission lines at all (Komossa \& Greiner 1999, Grupe et al. 1999, Gezari et al. 2003).

\subsubsection{Swift J1644+57}

Two events recently discovered with Swift, Swift J1644+57 and Swift J2058.4+0516, differ from previous flares, in the sense that they had much harder X-ray spectra, were accompanied by strong (beamed) radio emission, and exhibit some other remarkable properties (e.g., Burrows et al. 2011, Bloom et al. 2011, Zauderer et al. 2011, Levan et al. 2011, Cenko et al. 2012b). Swift 1644+57 was detected with Swift BAT in March 2011 and initially shared similarities with a GRB. However, the X-rays by far did not fade as quickly as expected for GRBs. The (isotropic) peak luminosity of the flare exceeded $10^{48} \mathrm{erg} / \mathrm{s}$. The X-ray lightcurve shows a general downward trend, on which rapid, high-amplitude variability is superposed, which can be as fast as 100 s. The host galaxy at redshift 0.35 does not show signs of permanent optical AGN activity. The event is accompanied by unresolved and variable radio emission, which has been interpreted as the rapid onset of a jet after stellar tidal disruption. Being extreme in its properties, and caught early in its evolution, this event has motivated a large number of follow-up observations and theoretical studies (see, e.g., the talks by Levan, Piran, Yu, Cenko, Zauderer, Metzger, Mimica, Castro-Tirado, Stone, and Shen at this workshop).

\subsection{X-ray TDE rates}

The RASS, the XMM-Newton slew survey, the Chandra deep fields, and clusters of galaxies, have all been used to estimate TDE rates in X-rays (Donley et al. 2002, Esquej et al. 2008, Luo et al. 2008, Maksym et al. 2010). All of these are on the order of $10^{-4}-10^{-5} / \mathrm{yr} /$ galaxy. They still come with significant uncertainties, and within these are well consistent with theoretical predictions (e.g., Merritt 2009, Brockamp et al. 2011).

\section{X-RAY TDES AS PROBES OF STRONG GRAVITY AND ACCRETION PHYSICS}

Upcoming X-ray surveys will detect more than 100 s or $1000 \mathrm{~s}$ of $\mathrm{TDEs}^{2}$, and many more will be identified in optical and radio transient surveys. Once followed up rapidly right after discovery, by deep X-ray observations, TDEs will provide us with new probes of strong gravity and accretion physics:

- Relativistic iron lines forming and evolving in the temporary accretion disk will probe GR.

- Precession effects map the Kerr metric.

- Emission-line profiles, lightcurves, and TDE rates can be used to measure or constrain black hole spin.

- The systematic spectral hardening of the TDE $\mathrm{X}$-ray spectra, first seen in ROSAT data of NGC 5905, may indicate the formation and evolution of a disk corona. X-ray spectroscopy can then be used to study the development of newly formed accretion-disk coronae.

\section{TDES AS SIGNPOSTS OF BINARY BHS, RECOILING BHS, INTERMEDIATE-MASS BHS, AND OTHER APPLICATIONS}

While the accretion physics near the event horizon, and GR effects, are best probed in $X$-rays, a number of other future

\footnotetext{
${ }^{2}$ Like the soft X-ray all-sky monitor $A S M$, a proposed mission on the Chinese space station dedicated to the study of TDEs and other transient events (Yuan et al. 2012).
} 


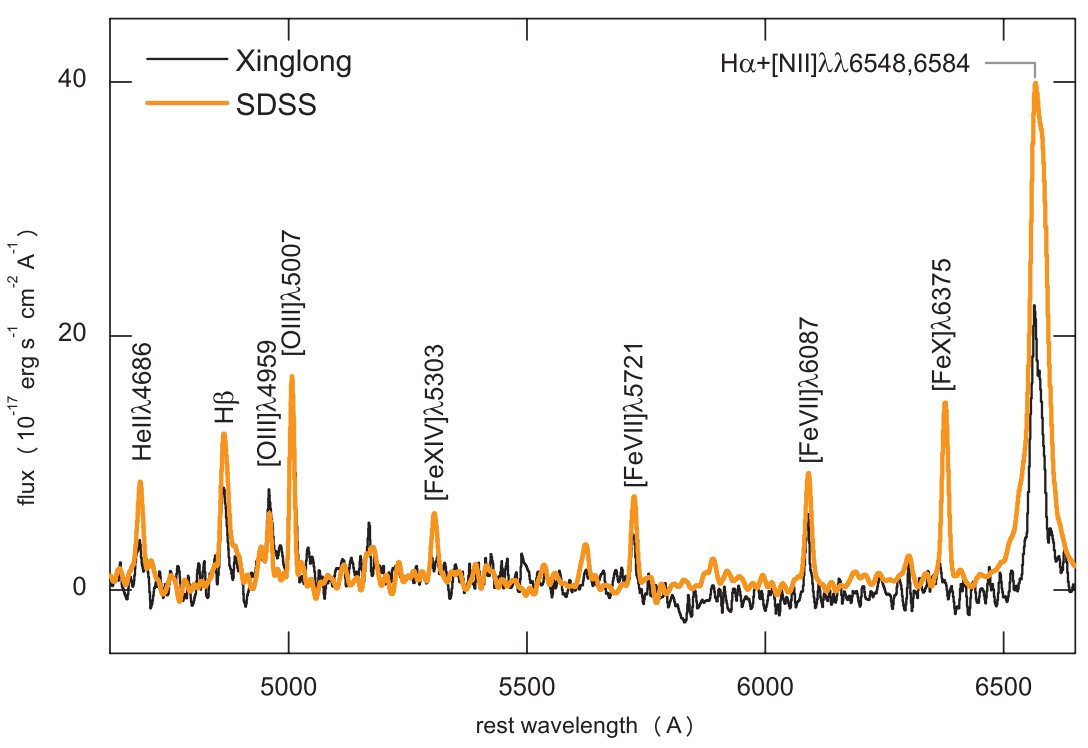

Figure 4. Super-strong and transient high-ionization emission lines in the optical spectrum of SDSSJ095209.56 +214313.3. Note that $[\mathrm{FeX}]$ is as strong as [OIII] in the SDSS high-state spectrum (orange). While the highest-ionization iron lines were very bright initially (orange), they were dramatically fainter in a Xinglong spectrum (black) taken several years later (see Komossa et al. 2008 for details). Note that the resolution of the SDSS spectrum was decreased for this plot, to match that of Xinglong.

applications of TDEs are interesting, no matter which waveband they are detected in.

- TDEs are markers of recoiling SMBHs. Tidal disruptions of stars in the star cluster bound to an ejected SMBH after GW recoil will produce off-center (or even intergalactic) flares of quasar-like luminosity, and in that way reveal the recoiling $\mathrm{BH}$ and its location (Komossa \& Merritt 2008). No mechanism other than TDEs produces off-nuclear transients as $\mathrm{X}$-ray luminous as quasars (GRBs are much more short-lived, supernovae much less X-ray luminous).

- TDEs are signposts of binary SMBHs. In the presence of a binary, TDE lightcurves show characteristic dips, caused by the temporary interruption of accretion (Liu et al. 2009).

- TDE rates depend on, and therefore probe, stellar dynamics in galaxy cores.

- TDEs will unveil the population of intermediate-mass black holes in the universe, if they exist.

- A small fraction of TDEs launches radio jets. These therefore provide us with an excellent opportunity of studying the early phase of formation and evolution of jets in systems which have been quiescent before. These events can be identified in upcoming radio transient surveys (e.g., Murphy et al. 2012).

\section{TDES IN AGN?}

Identifying TDEs in classical AGN is challenging, because the luminosity contrast between permanent accretion disk and temporary TDE flare will generally be low. Also, because in AGN with a permanent accretion disk, and with a large reservoir of obscuring material, there is a number of alternate mechanisms which can produce or mimik largeamplitude variability (e.g., Grupe et al. 2012a,b). Yet, TDEs are expected to occur in AGN, and disruption rates

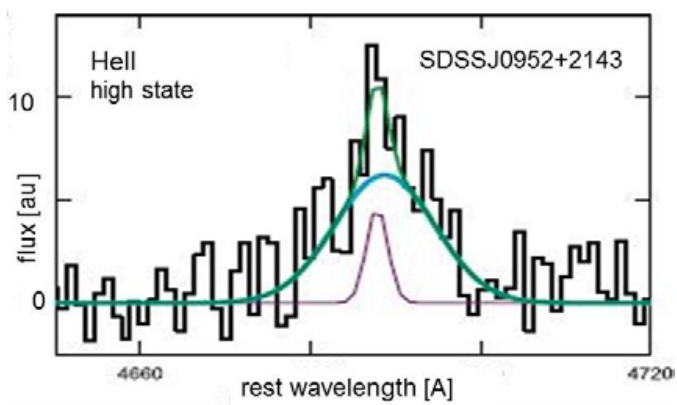

Figure 5. The HeII $\lambda 4686$ emission line of SDSSJ 095209.56+214313.3 during the 2005 SDSS highstate. It shows a broad profile (FWHM $=800 \mathrm{~km} / \mathrm{s})$ with a more narrow core. The emission line had faded significantly 3 years later.

may be even higher than in non-active galaxies (e.g., Karas \& Subr 2007). Especially, the AGN IC 3599 is remarkable for its high-amplitude X-ray outburst associated with a strong change in optical emission lines, and several mechanisms were considered to explain the data: highamplitude Narrow-line Seyfert 1 variability, variants of accretion-disk instabilities, or a TDE (Brandt et al. 1995, Grupe et al. 1995, Komossa \& Bade 1999).

In the future, with several well-observed X-ray TDEs in non-active galaxies now at hand, we can come back to the question of TDEs in AGN, using as a basis to identify them the observed X-ray properties of the events in the quiescent galaxies (X-ray softness, X-ray spectral evolution, and decline law, for instance).

\section{EMISSION-LINE REPROCESSING: IMAGINE WE COULD MAP A WHOLE GALAXY CORE, FOLLOWING ONE GIANT FLARE}

Tidal flares which occur in gas-rich galaxies will provide us with a powerful new tool of performing reverberation 
mapping of the cores of these galaxies. As the luminous electromagnetic radiation travels across the galaxy core, it will photoionize any circum-nuclear material (including the tidal debris itself) and is reprocessed into line radiation. This emission-line "light echo" enables us to perform "reverberation mapping" of any gaseous material that is present in the galaxy core. This will, in principle, include the broad-line region (if present), the molecular torus, and the interstellar medium (narrow-line region), and will also allow us to determine, which of these regions permanently exist in non-active galaxies. The emission-line fluxes, line widths, line shifts, and their evolution with time, tightly constrain the amount, density, composition, dynamics and geometry of the material.

Thanks to the Sloan Digital Sky Survey (SDSS; York et al. 2000) archive, and based on a systematic search, several candidate TDE emission-line reverberation signals have been identified in the last few years (Komossa et al. 2008, 2009, Wang et al. 2011, 2012; see Gezari et al. 2012 for the most recent such case, initially identified in the Pan-STARRS survey). The SDSS events are characterized by luminous, transient, high-ionization emission lines including transitions from [FeX]-[FeXIV], HeII, and Balmer lines of very unusual and variable profile (Fig. 4,5). Intrinsically luminous, the high-ionization lines fade dramatically on the timescale of years ${ }^{3}$. These lines have been excited by a luminous flare; yet the host galaxies of most of these events show little, if any, evidence for ongoing classical AGN activity. TDEs photoionizing circum-nuclear material, and perhaps the disrupted star itself, therefore provide a good explanation of the observed spectral features ${ }^{4}$.

Future detection of similar events, combined with dense spectroscopic sampling of the emission-line evolution, will provide us with a new means of reverberation-mapping the cores of (otherwise quiescent) galaxies, following one giant (tidal disruption) flare. Upcoming spectroscopic surveys like the one carried out with LAMOST will find many more emission-line signatures of TDEs.

\section{References}

[1] Amaro-Seoane, P., et al. 2007, CQGra, 24, 113

[2] Bade, N., et al. 1996, Astron. Astrophys., 309, L35

[3] Barres de Almeida, U., \& De Angelis, A. 2011, 2011arXiv1104.2528B

\footnotetext{
${ }^{3}$ Except for two sources which have constant line emission and so are likely AGN (T. Wang, priv. com.).

${ }^{4}$ See Komossa et al. (2009) and Wang et al. (2011, 2012) for an extended discussion of certain similarities, and other strong dissimilarities, with a small class of extreme supernovae of type IIn. Also note, that the broad and unusual HeII emission lines in two of these sources (Wang et al. 2011, Gezari et al. 2012) have been suggested to arise from the tidal debris of a Heliumrich stellar core. While the other events also show strongly variable HeII emission (Fig. 5 above; Komossa et al. 2008), HeII is accompanied by a wealth of other emission lines including Hydrogen.
}

[4] Bloom J.S., et al. 2011, Science, 33, 203

[5] Brandt, W.N., et al. 1985, MNRAS, 273, L47

[6] Brassart, M., \& Luminet, J.-P. 2010, Astron. Astrophys., 511, 80

[7] Brockamp, M., et al. 2011, MNRAS, 418, 1308

[8] Burrows, D.N., et al. 2011, Nature, 476, 421

[9] Cappelluti, N., et al. 2009, Astron. Astrophys., 495, 9

[10] Carter B., \& Luminet J.P., 1982, Nature, 296, 211

[11] Cenko, S.B., et al. 2012a, MNRAS, 420, 2684

[12] Cenko, S.B., et al. 2012b, ApJ, 753, 77

[13] Cheng, K.S., et al. 2006, ApJ, 645, 1138

[14] Donley, J.L., et al. 2002, AJ, 124, 1308

[15] Esquej, P., et al. 2007, Astron. Astrophys., 462, L49

[16] Esquej, P., et al. 2008, Astron. Astrophys., 489, 543

[17] Gezari, S., et al. 2003, ApJ, 592, 42

[18] Gezari, S., et al. 2006, ApJ, 653, L25

[19] Gezari, S., et al. 2012, Nature, 485, 217

[20] Gezari, S. 2012, these proceedings

[21] Greiner, J., et al. 2000, Astron. Astrophys., 362, L25

[22] Grupe, D., et al. 1995, Astron. Astrophys., 299, L5

[23] Grupe, D., et al. 1999, Astron. Astrophys., 350, L31

[24] Grupe, D., et al. 2012a, ApJS, 199, 28

[25] Grupe, D., et al. 2012b, these proceedings

[26] Guillochon, J., \& Ramirez-Ruiz, E. 2012, 2012arXiv1206.2350G

[27] Halpern, J., et al. 2004, ApJ, 604, 572

[28] Hayasaki, K., et al. 2012,2012arXiv1210.1333H

[29] Hills, J.G. 1975, Nature, 254, 295

[30] Karas, V., \& Subr, L. 2007, Astron. Astrophys., 470, 11

[31] Kesden, M. 2012, Phys. Rev. D., 86, 4026

[32] Khokhlov A., \& Melia F. 1996, ApJ, 457, L61

[33] Kim S.S., et al. 1999, ApJ, 519, 647

[34] Komossa, S. 2002, Reviews in Modern Astronomy, 15,27

[35] Komossa, S. 2004, IAUS, 222, 45, T. StorchiBergmann et al. (eds)

[36] Komossa, S., \& Bade, N. 1999, Astron. Astrophys., 343, 775

[37] Komossa, S., \& Greiner, J. 1999, Astron. Astrophys., 349, L45

[38] Komossa, S., \& Merritt, D. 2008, ApJ, 683, L21

[39] Komossa, S., et al. 2004, ApJ, 603, L17

[40] Komossa, S., et al. 2008, ApJ, 678, L13

[41] Komossa, S., et al. 2009, ApJ, 701, 105

[42] Levan, A.J., et al. 2011, Science, 33, 199

[43] Lin, D., et al. 2011, ApJ, 738, 52

[44] Liu, F., et al. 2009, ApJ, 706, L133

[45] Lodato, G., et al. 2009, MNRAS, 392, 332

[46] Lodato, G., \& Rossi, E.M. 2011, MNRAS, 410, 359

[47] Luminet, J.-P. 1985, Ann. Phys., 10, 101

[48] Luminet, J.-P., \& Marck, J.-A., 1985, MNRAS, 212, 57

[49] Luo, B., et al. 2008, ApJ, 674, 122

[50] Maksym, W.P., et al. 2010, ApJ, 722, 1035

[51] Merritt, D. 2009, ApJ, 694, 959

[52] Murphy, T., et al. 2012, 2012arXiv1207.1528M

[53] Rees, M.J. 1988, Nature, 33, 523

[54] Rees, M.J. 1990, Science, 247, 817

[55] Saxton, R.D., et al. 2012, Astron. Astrophys., 541, 106 
[56] Stone, N., et al. 2012, 2012arXiv1210.3374S

[57] Strubbe, L.E., \& Quataert, E. 2011, MNRAS, 415, 168

[58] Trümper, J. 2001, Encyclopedia of Astronomy and Astrophysics, Bristol: Institute of Physics Publishing

[59] van Velzen, S., et al. 2011a, ApJ, 741, 73

[60] van Velzen, S., et al. 2011b, MNRAS, 417, L51
[61] Wang, T., et al. 2011, ApJ, 740, 85

[62] Wang, T., et al. 2012, ApJ, 749, 115

[63] York, D.G., et al. 2000, AJ, 120, 1579

[64] Yuan, W., et al. 2012, An X-ray and UV all-sky monitor proposed for the Chinese Space Station, NAOC internal document

[65] Zauderer, B.A., et al. 2011, Nature, 476, 425 